\title{
CHIMICA DELLE SCUOLE SUPERIORI E ENEM: UN CONFRONTO CURRICULARE
}

\section{ARTICOLO ORIGINALE}

GORTZ, Julia Santana ${ }^{1}$, TATY, Salvador Rodrigues², FECURY, Amanda Alves ${ }^{3}$, DENDASCK, Carla Viana ${ }^{4}$, OLIVEIRA, Euzébio de ${ }^{5}$, DIAS, Claudio Alberto Gellis de Mattos $^{6}$

GORTZ, Julia Santana. Et al. Chimica delle scuole superiori e Enem: un confronto curriculare. Revista Científica Multidisciplinar Núcleo do Conhecimento. Anno 06, Ed.03, Vol.03, pagg. 89-99. Marzo 2021. ISSN: 2448-0959, Link di accesso: https://www.nucleodoconhecimento.com.br/formazione-it/confrontocurriculare, DOI: 10.32749/nucleodoconhecimento.com.br/formazione-it/confrontocurriculare

\section{ASTRATTO}

II Esame Nazionale di Scuola Superiore (ENEM) è una valutazione composta da un saggio e domande a scelta multipla. Questa valutazione mira a misurare le conoscenze acquisite durante il liceo. Lo scopo di questo articolo è confrontare il contenuto delle domande della componente Chimica dell'Esame Nazionale di Scuola Superiore (ENEM) tra gli anni dal 2014 al 2018 con il contenuto del curriculum del

\footnotetext{
${ }^{1}$ Studente del corso tecnico di chimica (scuola superiore) presso l'Istituto di educazione di base, tecnica e tecnologica di Amapá (IFAP).

${ }^{2}$ Chimico, Master in Chimica (UFMA), Professore e ricercatore nel Corso di Laurea in Chimica presso l'Istituto di Base, Tecnico e Tecnologico di Amapá (IFAP), Coordinatore del Corso Tecnico in Chimica (IFAP).

${ }^{3}$ Biomedico, PhD in Malattie Tropicali (UFPA), Professore e ricercatore del Corso di Medicina al Campus Macapá, Università Federale di Amapá (UNIFAP).

${ }^{4}$ Teologo, PhD in Psicoanalisi, ricercatore presso il Centro di Ricerca e Studi Avanzati - CEPA.

${ }^{5}$ Biologo, dottore di ricerca in malattie tropicali (UFPA), professore e ricercatore presso il corso di educazione fisica presso l'Università Federale del Pará (UFPA).

${ }^{6}$ Biologo, PhD in Teoria del comportamento e ricerca (UFPA), Professore e ricercatore del Corso di Laurea in Chimica presso I'Istituto di Base, Tecnico e Tecnologico di Amapá (IFAP) e del Corso di Laurea in Educazione Professionale e Tecnologica (PROFEPT IFAP).
}

RC: 78603

Disponível em: https://www.nucleodoconhecimento.com.br/formazione-it/confronto- 
corso di chimica tecnica presso l'Istituto Federale di Amapá (IFAP) . La ricerca è stata condotta utilizzando domande di chimica del Esame Nazionale di Scuola Superiore (ENEM) prese dal programma Super Professor (software). II contenuto insegnato nei tre anni del corso tecnico di chimica presso l'Istituto Federale di Amapá (IFAP) soddisfa i requisiti dell'Esame Nazionale di Scuola Superiore (ENEM). II carico di lavoro è sufficiente anche per lo sviluppo delle discipline di base e tecniche. L'analisi del contenuto dimostra che, trattandosi di un corso tecnico, fornisce una conoscenza approfondita, che aumenta la sovvenzione per la realizzazione dell'ENEM. Questo contenuto è formato dalla teoria e anche da una grande esperienza pratica (laboratorio). La conoscenza pratica aiuta enormemente la fissazione dell'apprendimento e fornisce la conoscenza per discutere i contenuti.

Parole chiave: insegnamento, chimica, ENEM, EPT, curriculum.

\section{INTRODUZIONE}

II Esame di maturità nazionale (ENEM) è una valutazione composta da un saggio e domande a scelta multipla. Le domande corrispondono alle aree delle scienze umane e delle sue tecnologie (storia, geografia, filosofia e sociologia I); Scienze naturali e loro tecnologie (fisica, chimica e biologia); Lingue, codici e loro tecnologie (portoghese, lingua straniera, arte e educazione fisica); e la matematica e le sue tecnologie (matematica). Questa valutazione mira a misurare le conoscenze acquisite durante il liceo (Moretto e Wittke, 2018).

Un istituto federale è un'istituzione con l'obiettivo di soddisfare la necessità di manodopera tecnica qualificata, in modo che gli studenti siano meglio preparati per acquisire sia le conoscenze di base che le conoscenze tecniche (Alves et al., 2013; Penha et al., 2020). Ci sono attualmente 38 istituti federali presenti in tutti gli stati (Brasile, 2018).

RC: 78603

Disponível em: https://www.nucleodoconhecimento.com.br/formazione-it/confronto- 
Nel 2007, il 25 ottobre, è stata creata la Scuola tecnica federale di Amapá (ETFAP). II 29 dicembre 2008, a causa della legge 11.892, ETFAP viene trasformata nell'Istituto federale di istruzione, scienza e tecnologia di Amapá (IFAP) (Brasile, 2019; Marques et al., 2020).

II suo target di riferimento è il $50 \%$ del livello di scuola media superiore, corso tecnico legato alla scuola superiore (integrato, successivo e concorrente); II 30\% dei diplomi di istruzione superiore, la laurea triennale e tecnologica e il $20 \%$ per i diplomi universitari. Con studi post-laurea: Lato Sensu e Stricto Sensu (Brasile, 2019; Castro et al., 2020).

II corso di chimica è stato aperto nel 2017, con 3 anni a tempo pieno. La sua funzione è quella di formare tecnici in grado di operare, controllare e monitorare i processi industriali e di laboratorio. Mantenere il controllo di qualità di materie prime, input e prodotti. Eseguire analisi di campioni, chimiche, chimico-fisiche e microbiologiche. Sviluppa processi e prodotti. I professionisti formati in questo corso hanno i seguenti campi di attività: industrie chimiche; Laboratori per controllo qualità, certificazione di prodotti chimici, alimentari e affini (Brasile, 2019a).

Nel liceo tecnico in chimica offerto dall'Istituto Federale di Amapá, il menu prevede il primo anno: chimica generale (chimica nella nostra vita quotidiana, evoluzione di modelli atomici e legami chimici, funzioni inorganiche; problemi ambientali e reazioni chimiche) e materiali tecnici (chimica sperimentale, chimica inorganica e chimica organica). Nel secondo anno si vedono: chimica generale (soluzioni, reazioni esotermiche ed endotermiche, velocità, fattori, equilibrio e spostamento ed elettrochimica) e questioni tecniche (chimico-fisico, chimica analitica, analisi strumentale e corrosione). Infine nel terzo anno: chimica generale (chimica organica, idrocarburi, funzioni ossigenate, azotate e miste e proprietà organiche) e materiali tecnici (petrolio e polimeri, processi chimici industriali e tecnologia dei biocarburanti) (Brasile, 2016).

RC: 78603

Disponível em: https://www.nucleodoconhecimento.com.br/formazione-it/confronto- 
Il contenuto delle domande di chimica di Enem è diviso in 10 unità principali, che sono: Trasformazioni chimiche (dove sono inclusi gli argomenti di numero atomico, numero di massa, isotopi, massa atomica, tavola periodica e reazioni chimiche); Rappresentazione delle trasformazioni chimiche (argomento che include il bilanciamento di equazioni chimiche e calcoli stechiometrici); Materiali, loro proprietà e usi (che include proprietà dei materiali, stati fisici dei materiali, cambiamenti di stato, miscele e forze intermolecolari); Acqua (che include soluzioni, acidi, basi, sali e ossidi, nomenclatura e indicatori); Trasformazioni chimiche ed energia (dove vengono inseriti contenuti come entalpia, equazioni termochimiche, legge di Hess, cella ed elettrolisi); Dinamica delle trasformazioni chimiche (che include velocità di reazione ed energia di attivazione); Trasformazione chimica ed equilibrio (che comprende i soggetti di equilibrio costante, equilibrio acido-base e $\mathrm{pH}$, solubilità salina e idrolisi); Composti del carbonio (include funzioni organiche, idrocarburi e polimeri); Rapporti della chimica con le tecnologie, la società e l'ambiente (include il tema dell'inquinamento); Energie chimiche nella vita di tutti i giorni (include i temi del petrolio, del gas naturale e del carbone) (Brasile, 2015).

\section{OBIETTIVI}

Confronta il contenuto delle domande della componente Chimica dell'Esame Nazionale di Scuola Superiore (ENEM) tra gli anni dal 2014 al 2018 con il contenuto del curriculum del corso di chimica tecnica presso l'Istituto Federale di Amapá (IFAP).

\section{METODOLOGIA}

La ricerca è stata condotta utilizzando domande di chimica del National High School Exam (ENEM) prese dal programma Super Professor (software), acquisito da Interbits Informática (https://www.sprweb.com.br/mod_superpro/index.php) . Nel programma sono state selezionate la disciplina chimica e gli anni dal 2014 al 2018. Le domande sono state classificate in base al programma. Successivamente, è stato

RC: 78603

Disponível em: https://www.nucleodoconhecimento.com.br/formazione-it/confronto- 
effettuato un confronto tra questi e il programma della componente chimica del corso tecnico presso I'Istituto Federale di Amapá (IFAP). La ricerca bibliografica è stata condotta su articoli scientifici, sui computer dell'Istituto presso I'Istituto Federale di Educazione, Scienza e Tecnologia di Amapá, Campus Macapá, situato a Rodovia BR 210 KM 3, s / n - Bairro Brasil Novo. CEP: 68.909-398, Macapá, Amapá, Brasile. I dati sono stati compilati nell'applicazione Excel, parte della suite Office di Microsoft Corporation.

\section{RISULTATI}

La tabella 1 mostra il contenuto delle domande di chimica ENEM tra il 2014 e il 2018, per quantità e percentuale di domande. Queste domande corrispondono al 78,82\% delle domande totali nel periodo. Domande su enzimi, carboidrati, indicatori, cambiamenti di stato, simboli, inquinamento e proprietà fisico-chimiche non compaiono nel periodo $(0,00 \%)$. Domande su struttura dell'atomo, cinetica chimica, densità, proprietà colligative, soluzioni, classificazione periodica, equazione ed equilibrio di reazione, funzioni inorganiche, pratiche di laboratorio, sostanza e miscela, catene di carbonio, isomeria piatta, olio e polimeri appaiono all'1,18\%. Le domande sulle concentrazioni della soluzione, l'equilibrio chimico o ionico, l'idrolisi del sale, il redox e le funzioni sono del 2,35\%. Le domande sui legami chimici e la geometria molecolare, la radioattività e l'isomeria spaziale corrispondono al 3,53\%. II calcolo stechiometrico e la separazione delle miscele corrispondono al $4,71 \%$ delle domande. Le domande sulla termochimica e sulle reazioni organiche sono del $7,06 \%$. Le forze intermolecolari ed elettrochimiche appaiono dell'8,24\%.

RC: 78603

Disponível em: https://www.nucleodoconhecimento.com.br/formazione-it/confronto- 
Tabella 1 Classificazione del contenuto delle domande di chimica ENEM tra il $2014 \mathrm{e}$ il 2018, per quantità e percentuale di domande.

\begin{tabular}{|c|c|c|}
\hline \multicolumn{3}{|c|}{ Classificaçăo do autor após analise de conteúdo } \\
\hline Conteúdo de Química & Quantidade & Porcentagem \\
\hline Enzimas & 0 & $0.00 \%$ \\
\hline Hidratos de Carbono & 0 & $0.00 \%$ \\
\hline Indicadores & 0 & $0.00 \%$ \\
\hline Mudanças de Estado & 0 & $0.00 \%$ \\
\hline Símbolos & 0 & $0.00 \%$ \\
\hline Poluição & 0 & $0.00 \%$ \\
\hline Proprieda des Físico-Químicas & 0 & $0.00 \%$ \\
\hline Es trutura do atomo & 1 & $1.18 \%$ \\
\hline Cinética Química & 1 & $1.18 \%$ \\
\hline Densidade & 1 & $1.18 \%$ \\
\hline Proprieda des Coligativas & 1 & $1.18 \%$ \\
\hline Soluções & 1 & $1.18 \%$ \\
\hline Class ificação Periódica & 1 & $1.18 \%$ \\
\hline Equacionamento e Balanceamento de Reações & 1 & $1.18 \%$ \\
\hline Funções Inorgânicas & 1 & $1.18 \%$ \\
\hline Práticas de Laboratório & 1 & $1.18 \%$ \\
\hline Substância e Mistura & 1 & $1.18 \%$ \\
\hline Cadeias Carbônicas & 1 & $1.18 \%$ \\
\hline Is omeria Plana & 1 & $1.18 \%$ \\
\hline Petróleo & 1 & $1.18 \%$ \\
\hline Polímeros & 1 & $1.18 \%$ \\
\hline Concentrações das Soluçôes & 2 & $2.35 \%$ \\
\hline Equilíbrio Químico ou lônico & 2 & $2.35 \%$ \\
\hline Hidrólise de Sa is & 2 & $2.35 \%$ \\
\hline Óxido-redução & 2 & $2.35 \%$ \\
\hline Funções & 2 & $2.35 \%$ \\
\hline Ligações Químicas e Geometria Molecular & 3 & $3.53 \%$ \\
\hline Radioatividade & 3 & $3.53 \%$ \\
\hline Isomeria Espacial & 3 & $3.53 \%$ \\
\hline Cálculo Estequiométrico & 4 & $4.71 \%$ \\
\hline Separação de Misturas & 4 & $4.71 \%$ \\
\hline Termoquímica & 6 & $7.06 \%$ \\
\hline Reações Orgânicas & 6 & $7.06 \%$ \\
\hline Forças intermoleculares & 7 & $8.24 \%$ \\
\hline Eletroquímica & 7 & $8.24 \%$ \\
\hline
\end{tabular}

RC: 78603

Disponível em: https://www.nucleodoconhecimento.com.br/formazione-it/confronto- 
La tabella 2 mostra il contenuto sovrapposto delle domande di chimica ENEM tra il 2014 e il 2018, per quantità e percentuale di domande. Le domande sovrapposte rappresentano il $21,18 \%$ del totale dell'esame, nel periodo. Le domande Forze intermolecolari + Proprietà fisico-chimiche, Forze intermolecolari + Proprietà colligative, Forze intermolecolari + Separazione di miscele, Legami chimici e geometria molecolare + Classificazione periodica, Idrolisi dei sali + Equazione ed equilibrio delle reazioni + Funzioni inorganiche, Bilancio chimico o ionico + Polimeri, carboidrati + riduzione dell'ossido, idrolisi dei sali + indicatori, simboli + sostanza e miscela, equazione ed equilibrio delle reazioni + inquinamento e inquinamento + catene di carbonio appaiono all'1,18\%. I problemi di Enzimi + Reazioni organiche, Concentrazioni di soluzioni + Soluzioni rappresentano il 2,35\%.

Tabella 2 Classificazione del contenuto sovrapposto delle domande di chimica ENEM tra il 2014 e il 2018, per quantità e percentuale di domande. Le domande sovrapposte rappresentano il $21,18 \%$

\begin{tabular}{l|c|c|}
\hline \multicolumn{3}{c}{ Classificação do autor após analise de conteúdo sobreposto } \\
\hline \multicolumn{1}{c|}{ Conteúdo de Química } & Quantidade & Porcentagem \\
\hline Forças intermoleculares + Propriedades Fisico-Quimicas & 1 & $1.18 \%$ \\
\hline Forças intermoleculares + propriedades coligativas & 1 & $1.18 \%$ \\
\hline Forças Intermoleculares + Separação de Misturas & 1 & $1.18 \%$ \\
\hline Forças Intermoleculares + Mudanças de Es tado & 1 & $1.18 \%$ \\
\hline Ligações Químicas e Geometria Molecular + Classificação Periódica & 1 & $1.18 \%$ \\
\hline Hidrólise de Sais + Equa cionamento e Balanceamento de Reações + & 1 & $1.18 \%$ \\
\hline Funções Inorgânicas & 1 & $1.18 \%$ \\
\hline Equilíbrio Químico ou lônico + Polímeros & 1 & $1.18 \%$ \\
\hline Hidratos de Carbono + Óxido-redução & 1 & $1.18 \%$ \\
\hline Hidrólise de Sais + Indicadores & 1 & $1.18 \%$ \\
\hline Simbolos + Substância e Mistura & 1 & $1.18 \%$ \\
\hline Equacionamento e Balanceamento de Reações + Poluição & 1 & $1.18 \%$ \\
\hline Poluição + Cadeias Carbônicas & 1 & $2.35 \%$ \\
\hline Enzimas + Reações Orgânicas & 2 & $2.35 \%$ \\
\hline Concentrações das Soluções + Soluções & 2 & $2.35 \%$ \\
\hline Cadeias Carbônicas + Reações Orgânicas & 2 & \\
\hline
\end{tabular}

RC: 78603

Disponível em: https://www.nucleodoconhecimento.com.br/formazione-it/confronto- 
Le materie del menu di chimica del corso tecnico di chimica dell'IFAP, per anno e unità (riferite a ciascun bimestre), sono riportate nella tabella 3. Nel corso di chimica, le materie sono suddivise tra scuola media normale e tecnica materie specifiche del corso. Nelle discipline del curriculum standard, la componente utilizzata nel triennio è la chimica generale. II suo carico di lavoro totale è di 240 ore, 80 ore all'anno. Nel primo anno la tua prima unità è data in 15 ore, la seconda unità in 20 ore, la terza in 25 ore e la quarta in 20 ore. Nel secondo anno, il primo contenuto viene fornito in 15 ore, il secondo contenuto in 15 ore, il terzo in 25 ore e il quarto in 25 ore. Nel terzo anno, la prima unità ha un carico di lavoro di 15 ore, la seconda unità ha un carico di lavoro di 20 ore, la terza di 30 ore e la quarta di 15 ore.

Le altre materie (curriculari tecnici) indicate nella tabella 3 sono materie tecniche specifiche del corso, sono semestrali e hanno un carico di lavoro complessivo di 80 ore a semestre ciascuna. Nel primo anno vengono citate le materie: chimica sperimentale, che ha un carico di lavoro di 10 ore, 20 ore, 25 ore e 25 ore rispettivamente per le unità uno, due, tre e quattro; chimica inorganica, con un carico di lavoro di 15 ore per la prima unità, 15 ore per la seconda unità, 25 ore per la terza unità e 25 ore per la quarta unità; chimica organica, la cui prima unità è data in 25 ore, la seconda unità in 15 ore, la terza in 20 ore e la quarta in 20 ore. Nel secondo anno le materie tecniche sono le seguenti: Fisico-chimica, con la prima unità impartita in 25 ore, la seconda unità in 20 ore, la terza in 20 ore e la quarta in 15 ore; chimica analitica, con 18 ore per la prima unità, 20 ore per la seconda unità, 23 ore per la terza unità e 19 ore per la quarta unità; l'analisi strumentale, con le sue quattro unità, è data rispettivamente in 10 ore, 20 ore, 25 ore e 25 ore; corrosione, dove la tua prima unità è data in 10 ore, la tua seconda unità è data in 25 ore, la tua terza unità è data in 20 ore e la tua quarta in 25 ore. Nel terzo anno le discipline specifiche indicate in tabella sono: olio e polimeri, con le loro unità espresse rispettivamente in 18 ore, 22 ore, 22 ore e 18 ore; processi chimici industriali, con la prima unità data in 25 ore, la seconda unità in 20 ore, la terza unità in 20 ore e la quarta in 15 ore;

$\mathrm{RC}: 78603$

Disponível em: https://www.nucleodoconhecimento.com.br/formazione-it/confronto- 
tecnologia dei biocarburanti, con un carico di lavoro di 25 ore per la prima unità, 25 ore per la seconda unità, 10 ore per la terza e 20 ore per la quarta.

RC: 78603

Disponível em: https://www.nucleodoconhecimento.com.br/formazione-it/confrontocurriculare 
Tabella 3. Materie del menu di chimica del corso tecnico di chimica all'IFAP, per anno e unità.

DISAPLINA CURRICULAR PADRĀO

\begin{tabular}{|c|c|c|c|c|c|c|}
\hline \multirow[b]{2}{*}{ Unidade } & \multicolumn{6}{|c|}{ Ano } \\
\hline & $1^{\circ} \mathrm{ANO}$ & $\begin{array}{c}\mathbf{n}^{\circ} \text { de } \\
\text { horas aula }\end{array}$ & $2^{\circ} \mathrm{ANO}$ & de horas at & $3^{\circ} \mathrm{ANO}$ & $\begin{array}{c}\mathbf{n}^{\circ} \text { de } \\
\text { horas aula }\end{array}$ \\
\hline I & $\begin{array}{c}\text { A Quimica emnosso } \\
\text { cotidiano }\end{array}$ & 15 horas & Solbções & 15 horas & Quimica Orgânica & 15 horas \\
\hline II & $\begin{array}{l}\text { A evobção dos } \\
\text { modelos atômicos e } \\
\text { ligacões Qvímic as. }\end{array}$ & 20 horas & $\begin{array}{c}\text { Reações exotémicas } \\
\text { e endotérnicas }\end{array}$ & 15 horas & Hidrocarb onetos & 20 horas \\
\hline III & $\begin{array}{c}\text { Funções Inorgânicas } \\
\text { e problemas } \\
\text { anbientais }\end{array}$ & 25 horas & $\begin{array}{c}\text { Velocidiade, fatores, } \\
\text { equilibrio e } \\
\text { deslocamento }\end{array}$ & 25 horas & $\begin{array}{l}\text { Funções Oxigenadas, } \\
\text { nitrogenadas e mis tas }\end{array}$ & 30 horas \\
\hline IV & Reações Quimicas & 20 horas & Eletro-Quimica & 25 horas & $\begin{array}{c}\text { Propriedades } \\
\text { Orgânicas }\end{array}$ & 15 horas \\
\hline & & 80 horas & & 80 horas & & 80 horas \\
\hline
\end{tabular}

DISCIPLINA CURRICULAR TÉCNICA - 1 ANO

\begin{tabular}{|c|c|c|c|c|c|c|}
\hline \multirow[b]{2}{*}{ Unidade } & \multicolumn{6}{|c|}{ Ano } \\
\hline & $\begin{array}{c}\text { Quimica } \\
\text { Experimental }\end{array}$ & $\begin{array}{c}\mathbf{n}^{\circ} \text { de } \\
\text { horas aula }\end{array}$ & $\begin{array}{l}\text { Quimica } \\
\text { Inorgânica }\end{array}$ & $\begin{array}{c}\mathbf{n}^{\circ} \text { de } \\
\text { horas aula }\end{array}$ & $\begin{array}{l}\text { Quimica } \\
\text { Orgânica }\end{array}$ & $\begin{array}{c}\mathbf{n}^{\circ} \mathrm{de} \\
\text { horas aula }\end{array}$ \\
\hline I & $\begin{array}{c}\text { Introdução a os } \\
\text { trabalhos em } \\
\text { laboratónio. }\end{array}$ & 10 horas & $\begin{array}{l}\text { Quimica dos não } \\
\text { metais }\end{array}$ & 15 horas & $\begin{array}{c}\text { Introdução a } \\
\text { macanismo de reação }\end{array}$ & 25 horas \\
\hline II & $\begin{array}{c}\text { Obtenção e us o de } \\
\text { calor }\end{array}$ & 20 horas & Quimica dos metais & 15 horas & Ácidos e Bases & 15 horas \\
\hline III & $\begin{array}{c}\text { Process os de } \\
\text { separação de mis turas }\end{array}$ & 25 horas & $\begin{array}{l}\text { Compostos de } \\
\text { coordenação }\end{array}$ & 25 horas & $\begin{array}{c}\text { Reações de adição- } \\
\text { eliminação } \\
\text { nucleofilica em } \\
\text { carbono acilico }\end{array}$ & 20 horas \\
\hline IV & $\begin{array}{c}\text { Estudo das reações } \\
\text { Quimicas }\end{array}$ & 25 horas & $\begin{array}{l}\text { Estrutura de sólidos } \\
\text { cristalinos e amorfos }\end{array}$ & 25 horas & $\begin{array}{c}\text { Reações de } \\
\text { subs tituição } \\
\text { eletrofilica em } \\
\text { aromáticos }\end{array}$ & 20 horas \\
\hline & & 80 horas & & 80 horas & & 80 horas \\
\hline
\end{tabular}

\begin{tabular}{|c|c|c|c|c|c|c|c|c|}
\hline \multirow[b]{2}{*}{ Unidade } & \multicolumn{8}{|c|}{ Ano } \\
\hline & Fisico Quimira & $\begin{array}{c}\mathbf{n}^{\circ} \text { de } \\
\text { horas aula }\end{array}$ & Quimica Analítica & $\begin{array}{c}\mathbf{n}^{\circ} \text { de } \\
\text { horas aula }\end{array}$ & $\begin{array}{c}\text { Análise } \\
\text { Instrumental }\end{array}$ & $\begin{array}{c}\mathrm{n}^{\circ} \text { de } \\
\text { horas aula }\end{array}$ & Corrosão & $\begin{array}{c}\mathrm{n}^{\circ} \text { de horas } \\
\text { aula }\end{array}$ \\
\hline I & $\begin{array}{c}\text { Solvções, Dispersões } \\
\text { e Propriadades } \\
\text { Coligativas }\end{array}$ & 25 horas & $\begin{array}{c}\text { Introdução, Tecnic as } \\
\text { e Métodos }\end{array}$ & 18 horas & $\begin{array}{c}\text { Introdução a aná lise } \\
\text { instrumental }\end{array}$ & 10 horas & Introd vção a corro são & 10 horas \\
\hline II & Equilibrio Quimico & 20 horas & $\begin{array}{c}\text { Titulometria de } \\
\text { neutralizaça âoe } \\
\text { precipitação }\end{array}$ & 20 horas & $\begin{array}{c}\text { Mítodos } \\
\text { eletroanaliticos }\end{array}$ & 20 horas & $\begin{array}{c}\text { Princ ipio s básicos da } \\
\text { corrosảo } \\
\text { eletroQuimica }\end{array}$ & 25 horas \\
\hline III & Cinética Química & 20 horas & $\begin{array}{c}\text { Titulometria de } \\
\text { complexaça âo e oxi- } \\
\text { reducăa }\end{array}$ & 23 horas & $\begin{array}{c}\text { Métodos } \\
\text { espectros cópicos }\end{array}$ & 25 horas & $\begin{array}{c}\text { Princ ípios básicos da } \\
\text { corrosảo Química }\end{array}$ & 20 horas \\
\hline IV & $\begin{array}{c}\text { Es tudo dos } \\
\text { Proces sos de Troca } \\
\text { de Calor nos } \\
\text { Equilibrios }\end{array}$ & 15 horas & Laboratóio, Caku los & 19 horas & $\begin{array}{c}\text { Métodos } \\
\text { cromatográficos }\end{array}$ & 25 horas & $\begin{array}{l}\text { Res istêncicia à } \\
\text { corros åo e proteçăo } \\
\text { an ticorro siva }\end{array}$ & 25 horas \\
\hline & & 80 horas & & 80 horas & & 80 horas & & 80 horas \\
\hline
\end{tabular}

RC: 78603

Disponível em: https://www.nucleodoconhecimento.com.br/formazione-it/confronto- 
DISAPLINA CURRICULAR PADRĀO

\begin{tabular}{|c|c|c|c|c|c|c|}
\hline \multirow[b]{2}{*}{ Unidade } & \multicolumn{6}{|c|}{ Ano } \\
\hline & $1^{\circ}$ ANO & $\begin{array}{c}\mathbf{n}^{\circ} \text { de } \\
\text { horas aula }\end{array}$ & $2^{\circ}$ ANO & de horas al & $3^{\circ}$ ANO & $\begin{array}{c}\mathbf{n}^{\circ} \text { de } \\
\text { horas aula }\end{array}$ \\
\hline I & $\begin{array}{c}\text { A. Quimica emnosso } \\
\text { cotidiano }\end{array}$ & 15 horas & Solbções & 15 horas & Química Orgânica & 15 horas \\
\hline II & $\begin{array}{l}\text { A evohção dos } \\
\text { modelos atômicos e } \\
\text { ligações Qvimicas. }\end{array}$ & 20 horas & $\begin{array}{l}\text { Reações exotérmicas } \\
\text { e endotérmicas }\end{array}$ & 15 horas & Hidrocarb onetos & 20 horas \\
\hline III & $\begin{array}{c}\text { Funções Inorgânicas } \\
\text { e problemas } \\
\text { ambientais }\end{array}$ & 25 horas & $\begin{array}{c}\text { Velocidiade, fatores, } \\
\text { equilibrio e } \\
\text { deslocamento }\end{array}$ & 25 horas & $\begin{array}{l}\text { Funções Oxigenadas, } \\
\text { nitrogenadas e mis tas }\end{array}$ & 30 horas \\
\hline IV & Reações Quimicas & 20 horas & Eletro-Quimica & 25 horas & $\begin{array}{c}\text { Propriedades } \\
\text { Orgânicas }\end{array}$ & 15 horas \\
\hline & & 80 horas & & 80 horas & & 80 horas \\
\hline
\end{tabular}

DISCIPLINA CURRICULAR TÉCNICA - 10 ANO

\begin{tabular}{|c|c|c|c|c|c|c|}
\hline \multirow[b]{2}{*}{ Unidade } & \multicolumn{6}{|c|}{ Ano } \\
\hline & $\begin{array}{c}\text { Quimica } \\
\text { Experimental }\end{array}$ & $\begin{array}{c}\mathbf{n}^{\circ} \text { de } \\
\text { horas aula }\end{array}$ & $\begin{array}{l}\text { Quimica } \\
\text { Inorgânica }\end{array}$ & $\begin{array}{c}\mathbf{n}^{\circ} \text { de } \\
\text { horas aula }\end{array}$ & $\begin{array}{l}\text { Quimica } \\
\text { Orgânica }\end{array}$ & $\begin{array}{c}\mathbf{n}^{\circ} \text { de } \\
\text { horas aula }\end{array}$ \\
\hline I & $\begin{array}{l}\text { Introdução a os } \\
\text { trabalhos em } \\
\text { laboratónio. }\end{array}$ & 10 horas & $\begin{array}{l}\text { Quimica dos não } \\
\text { metais }\end{array}$ & 15 horas & $\begin{array}{c}\text { Introdução a } \\
\text { mecanismo de reação }\end{array}$ & 25 horas \\
\hline II & $\begin{array}{c}\text { Obtenção e us o de } \\
\text { calor }\end{array}$ & 20 horas & Quimica dos metais & 15 horas & Ácidos e Bases & 15 horas \\
\hline III & $\begin{array}{c}\text { Process os de } \\
\text { separação de mis turas }\end{array}$ & 25 horas & $\begin{array}{l}\text { Compostos de } \\
\text { coordenação }\end{array}$ & 25 horas & $\begin{array}{c}\text { Reações de adição- } \\
\text { eliminação } \\
\text { nucleofilica em } \\
\text { carbono acilico }\end{array}$ & 20 horas \\
\hline IV & $\begin{array}{c}\text { Estudo das reações } \\
\text { Quimicas }\end{array}$ & 25 horas & $\begin{array}{l}\text { Estrutura de sólidos } \\
\text { cristalinos e amorfos }\end{array}$ & 25 horas & $\begin{array}{l}\text { Reações de } \\
\text { substituição } \\
\text { eletrofilica em } \\
\text { aromáticos }\end{array}$ & 20 horas \\
\hline & & 80 horas & & 80 horas & & 80 horas \\
\hline
\end{tabular}

DISAPLINA CURRICULAR TÉCNICA - 2\% ANO

\begin{tabular}{|c|c|c|c|c|c|c|c|c|}
\hline \multirow[b]{2}{*}{ Unidade } & \multicolumn{8}{|c|}{ Ano } \\
\hline & Fisico Quimira & $\begin{array}{c}\mathbf{n}^{\circ} \text { de } \\
\text { horas aula }\end{array}$ & Quimica Analítica & $\begin{array}{c}\mathrm{n}^{\circ} \mathrm{de} \\
\text { horas aula }\end{array}$ & $\begin{array}{c}\text { Análise } \\
\text { Instrumental }\end{array}$ & $\begin{array}{c}\mathbf{n}^{\circ} \text { de } \\
\text { horas aula }\end{array}$ & Corrosão & $\begin{array}{c}n^{\circ} \text { de horas } \\
\text { aula }\end{array}$ \\
\hline I & $\begin{array}{c}\text { Solvções, Dispersões } \\
\text { e Proprigdades } \\
\text { Coligativas }\end{array}$ & 25 horas & $\begin{array}{c}\text { Introdução, Tecric as } \\
\text { e Métodos }\end{array}$ & 18 horas & $\begin{array}{l}\text { Introdução a análise } \\
\text { instrumental }\end{array}$ & 10 horas & Introd vção a corro são & 10 horas \\
\hline II & Equilibrio Quimico & 20 horas & $\begin{array}{l}\text { Titulometria de } \\
\text { neutralizaça ào e } \\
\text { precipitação }\end{array}$ & 20 horas & $\begin{array}{c}\text { Métodos } \\
\text { ele troan aliticos }\end{array}$ & 20 horas & $\begin{array}{c}\text { Princ ípios básicos da } \\
\text { corrosảo } \\
\text { eletroQuímica }\end{array}$ & 25 horas \\
\hline III & Cinética Química & 20 horas & $\begin{array}{c}\text { Titulometria de } \\
\text { complexaçäa e oxi- } \\
\text { redução }\end{array}$ & 23 horas & $\begin{array}{c}\text { Métodos } \\
\text { espectros cóp iros }\end{array}$ & 25 horas & $\begin{array}{l}\text { Principios básicos da } \\
\text { corrosảo Química }\end{array}$ & 20 horas \\
\hline IV & $\begin{array}{c}\text { Es tudo dos } \\
\text { Processos de Troca } \\
\text { de Calor nos } \\
\text { Equilibrios }\end{array}$ & 15 horas & Laboratócio, Cácu los & 19 horas & $\begin{array}{c}\text { Métodos } \\
\text { crome tográficos }\end{array}$ & 25 horas & $\begin{array}{l}\text { Resistência à } \\
\text { corros ào e proteção } \\
\text { anticorro siva }\end{array}$ & 25 horas \\
\hline & & 80 horas & & 80 horas & & 80 horas & & 80 horas \\
\hline
\end{tabular}

RC: 78603

Disponível em: https://www.nucleodoconhecimento.com.br/formazione-it/confronto- 
DISCIPLINA CURRICULAR TÉCNICA - 3 ANO

\begin{tabular}{|c|c|c|c|c|c|c|}
\hline \multirow[b]{2}{*}{ Unidade } & \multicolumn{6}{|c|}{ Ano } \\
\hline & $\begin{array}{l}\text { Petróleo e } \\
\text { Polimeros }\end{array}$ & $\begin{array}{c}\mathbf{n}^{\circ} \text { de } \\
\text { horas aula }\end{array}$ & $\begin{array}{l}\text { Processos } \\
\text { Qúmicos } \\
\text { Industriais }\end{array}$ & $\begin{array}{c}\mathbf{n}^{\circ} \text { de } \\
\text { horas aula }\end{array}$ & $\begin{array}{c}\text { Tecnologia de } \\
\text { Biocombustiveis }\end{array}$ & $\begin{array}{c}\mathbf{n}^{\circ} \mathrm{de} \\
\text { horas aula }\end{array}$ \\
\hline $\mathbf{I}$ & $\begin{array}{c}\text { Process os } \\
\text { tecnológicos de } \\
\text { petróleo e polimeros e } \\
\text { Legislação pertinente }\end{array}$ & 18 horas & $\begin{array}{l}\text { Tratamento de água, } \\
\text { Importancia do } \\
\text { Tratamento de } \\
\text { Efluentes, Parâmetros } \\
\text { de pohrição hidrica e } \\
\text { Classificação de } \\
\text { residuos }\end{array}$ & 25 horas & $\begin{array}{l}\text { Etapas Quimicas da } \\
\text { Sintese de Etanol e do } \\
\text { Biodiesel; Análise } \\
\text { Quimica de Qualidade } \\
\text { de Produção de Mini - } \\
\text { Usinas Pequeno, } \\
\text { Médio e Grande Porte }\end{array}$ & 25 horas \\
\hline II & $\begin{array}{l}\text { Controle de qualidade } \\
\text { e Fundamentos do } \\
\text { petróleo e dos } \\
\text { polimeros }\end{array}$ & 22 horas & $\begin{array}{c}\text { Tratamento e } \\
\text { disposição final de } \\
\text { efluentes de } \\
\text { residvo, Formas de } \\
\text { tratamentos, Tipos de } \\
\text { tratamento e descarte } \\
\text { e Tpos de } \\
\text { equipamentos }\end{array}$ & 20 horas & $\begin{array}{c}\text { Produção e Análise } \\
\text { Quimica de } \\
\text { Biocombus tiveis em } \\
\text { Escala Laboratoriale } \\
\text { Industrial }\end{array}$ & 25 horas \\
\hline III & $\begin{array}{l}\text { Indús tria do petróleo } \\
\text { e petroQvimica e } \\
\text { Logistica do petróleo }\end{array}$ & 22 horas & $\begin{array}{c}\text { Programas de } \\
\text { reutilização, } \\
\text { Resolução de } \\
\text { problemas de } \\
\text { produção e } \\
\text { qualidade de } \\
\text { alimentos, Otimiac ção } \\
\text { na produção de } \\
\text { oleaginos as e açúcar }\end{array}$ & 20 horas & $\begin{array}{l}\text { Produção de Bio- } \\
\text { Etanol e Biodiesel }\end{array}$ & 10 horas \\
\hline IV & $\begin{array}{l}\text { Produção de } \\
\text { polimeros e } \\
\text { Reciclagemde } \\
\text { polimeros }\end{array}$ & 18 horas & $\begin{array}{c}\text { Otimização na } \\
\text { produção de álcool, } \\
\text { Oleaginos as da regão } \\
\text { e Reações Quimicas } \\
\text { dos mais importantes } \\
\text { processos industriais }\end{array}$ & 15 horas & $\begin{array}{l}\text { Aspectos } \\
\text { operacionais de } \\
\text { usinas de etanol } \\
\text { biodiesele, } \\
\text { combus tiveis de } \\
\text { Terceira geração }\end{array}$ & 20 horas \\
\hline & & 80 horas & & 80 horas & & 80 horas \\
\hline
\end{tabular}

La somma delle domande di chimica ENEM tra il 2014 e il 2018, per grado di difficoltà, per anno appare nella figura 1. II numero di domande con difficoltà media è aumentato da sette (7) nel 2014 a dodici (12) nel 2015. In entrambi gli anni successivi c'è stata una riduzione, con sette (7) domande nel 2016 e cinque (5) nel 2017. Nel 2018 c'è stato un piccolo aumento, per un totale di sei (6) domande. In materia di elevata complessità, c'è stata una riduzione da dieci (10) domande nel 2014 a cinque (5) nel 2015. Da allora in poi c'è stato un aumento nei due anni

RC: 78603

Disponível em: https://www.nucleodoconhecimento.com.br/formazione-it/confronto- 
successivi, con dieci (10) nel 2016 e dodici (12) nel 2017 Nel 2018 c'è stato un piccolo calo nel numero, undici (11) domande).

La figura 1 mostra la somma delle domande di chimica ENEM tra il 2014 e il 2018, per grado di difficoltà, per anno

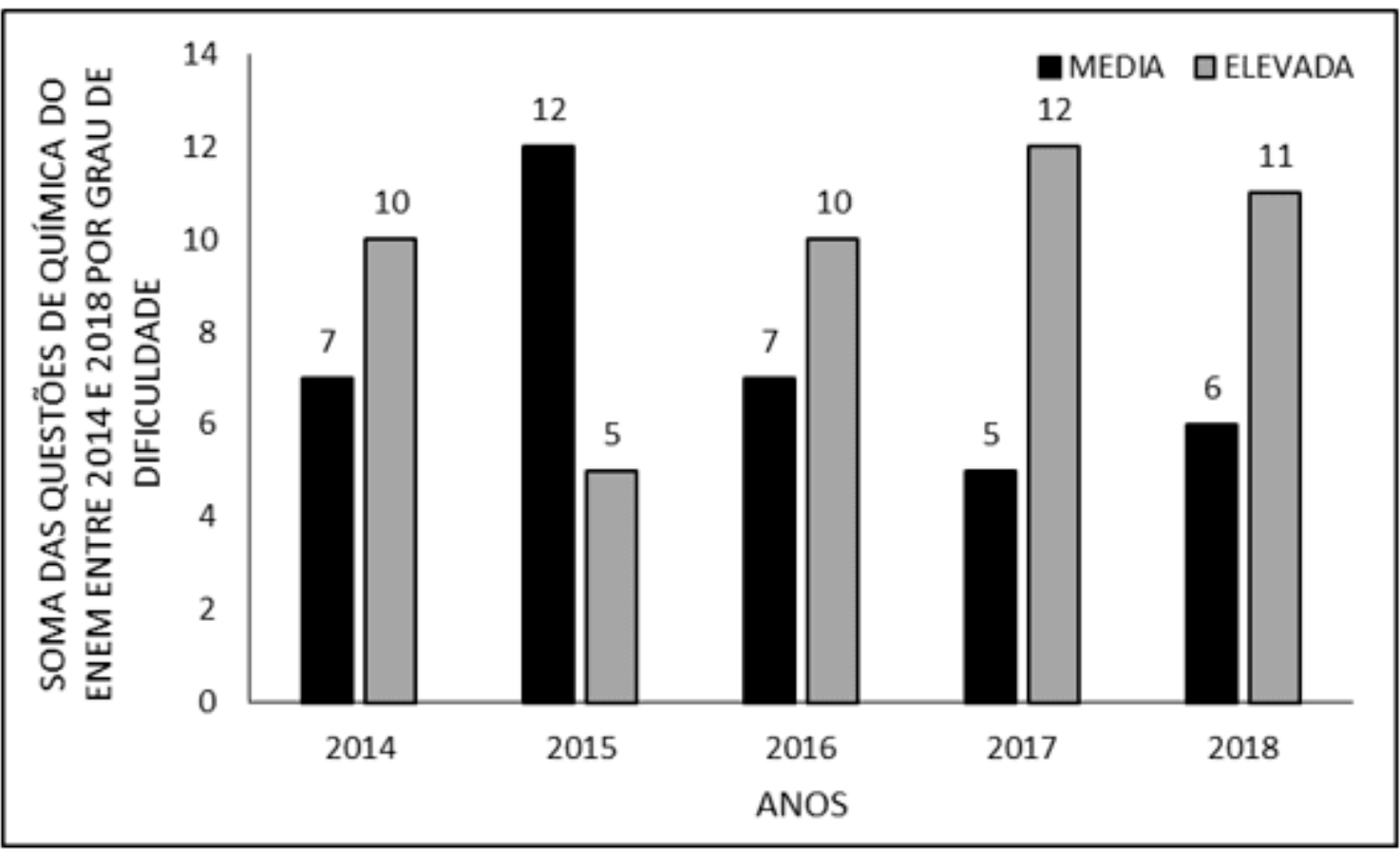

\section{DISCUSSIONE}

La differenza presente nei test ENEM valutati, quanto alla percentuale di ciascun contenuto (Tabella 1), si verifica probabilmente perché alcuni di essi sono più presenti nella vita quotidiana degli studenti (come le reazioni organiche e i legami tra loro) rispetto ad altri ( indicatori, cambiamenti di stato, simbologia). ENEM valuta per contestualizzare le sue domande (Hipólito e Silveira, 2011; Silveira et al., 2014).

I contenuti di ENEM si sovrappongono perché cercano un'integrazione tra le tematiche interne della matrice curricolare di chimica e tra queste tematiche e le altre discipline (interdisciplinarietà e trasversalità) (Tabella 2). Questa miscela di contenuti

$\mathrm{RC}: 78603$

Disponível em: https://www.nucleodoconhecimento.com.br/formazione-it/confronto- 
li aiuta ad avvicinarsi alla vita quotidiana degli studenti. Aiuta anche a cambiare il curriculum in qualcosa di meno intonacato e più trasversale (Hipólito e Silveira, 2011).

Questa tabella mostra anche che la maggior parte delle domande intradisplinarie riguardano enzimi, reazioni organiche, soluzioni e concentrazione di soluzioni. Ciò è probabilmente dovuto al fatto che, fisiologicamente, la maggior parte degli esseri viventi funziona grazie alla chimica della loro struttura organica. Quindi, ancora una volta, domande come questa sono più vicine alla vita quotidiana degli studenti (Leite e Velani, 2019).

I contenuti possono avere carichi di lavoro diversi (Tabella 3) a causa delle differenze di complessità e anche della capacità di contestualizzare il contenuto. Contestualizzare il contenuto è importante per la loro comprensione e comprensione. Poiché ogni insegnante ha il proprio modo unico di insegnargli, potrebbe esserci una differenza nel tempo del curriculum programmato nella pianificazione con il tempo reale per insegnargli (Pontes et al., 2008).

L'area delle scienze naturali ha il peso maggiore per l'area sanitaria e biologica nella maggior parte delle università pubbliche del Brasile, come USP, una delle università più prestigiose del paese. I corsi in ambito sanitario sono molto ambiti, soprattutto per la loro remunerazione. Le domande di chimica probabilmente cercano di mantenere una maggiore difficoltà (Figura 1 ) in modo che i candidati con più conoscenze in quest'area di grande importanza per il corso entrino con più conoscenza in queste (Casoni, 2021; Ufpe, 2021).

\section{CONCLUSIONE}

II contenuto insegnato nei tre anni del corso tecnico di chimica presso l'Istituto Federale di Amapá (IFAP) soddisfa i requisiti dell'Esame Nazionale di Scuola Superiore (ENEM).

RC: 78603

Disponível em: https://www.nucleodoconhecimento.com.br/formazione-it/confronto- 
Il carico di lavoro del corso tecnico di chimica della scuola superiore presso IFAP è anche sufficiente per lo sviluppo di discipline tecniche e di base e fornisce anche una buona base per ENEM

L'analisi dei contenuti del corso ha chimica da IFAP dimostra che, trattandosi di un corso tecnico, fornisce una conoscenza approfondita, che aumenta il sussidio per il completamento di ENEM. Questo contenuto è formato dalla teoria e anche da una grande esperienza pratica (laboratorio). La conoscenza pratica aiuta enormemente la fissazione dell'apprendimento e fornisce la conoscenza per discutere i contenuti.

Pertanto, il contenuto curriculare di questo corso tecnico soddisfa i requisiti di base dell'Esame Nazionale.

\section{RIFERIMENTI}

ALVES, N. B.; PALMA, L. C.; SILVA, T. N. Educação para a sustentabilidade: a construção de caminhos no Instituto Federal de Educação, Ciência e Tecnologia do Rio Grande do Sul (IFRS). Ram, Rev. Adm. Mackenzie, v. 14, n. 3, p. 83-118, 2013.

BRASIL. Matriz de Referência Enem. Brasilia DF, 2015. Disponível em: < http://download.inep.gov.br/download/enem/matriz_referencia.pdf >. Acesso em: 25 mar 2020.

. Curso Técnico de Nível Médio em Química na Forma Integrada Regime Integral: Plano de Curso. Macapá AP, 2016. Disponível em: < https://portal.ifap.edu.br/index.php/publicacoes/item/1100-resolucao-n-20-2018consup $>$.

. Expansão da Rede Federal. Rede Federal de Educação Profissional, Científica e Tecnológica. 2018. Disponível em: < http://redefederal.mec.gov.br/expansao-da-rede-federal >. Acesso em: 20 abr 2020.

RC: 78603

Disponível em: https://www.nucleodoconhecimento.com.br/formazione-it/confronto- 
Histórico. Macapá AP, 2019. Disponível em: < http://portal.ifap.edu.br/index.php/quem-somos/historico >. Acesso em: 24 abr. 2020. Curso Técnico em Química - Integrado - Campus Macapá. Macapá AP, 2019a. Disponível em: http://www.ifap.edu.br/index.php/component/content/article?id=398 >. Acesso em: 24 abr. 2020.

CASONI, L. A. SiSU Simulator. 2021. Disponível em: < https://sisusimulator.com.br/usp/faculdade-de-medicina-da-universidade-de-saopaulo-fm/medicina-bacharelado >. Acesso em: 24 fev 2021

CASTRO, G. N. V. et al. Análise de Eficiência Acadêmica dos cursos subsequentes, nas modalidades à distância e presencial, ofertados pelo Instituto Federal do Amapá (2018). Research, Society and Development, v. 9, n. 8, p. e208985262, 2020. https://rsdjournal.org/index.php/rsd/article/view/5262

HIPÓLITO, A. F.; SILVEIRA, H. E. D. As questões de Química do Exame Nacional do Ensino Médio (ENEM) em um enfoque transversal e interdisciplinar. 2011. Disponível em: < http://abrapecnet.org.br/atas_enpec/viiienpec/resumos/R0237-1.pdf >. Acesso em: 11 fev 2021.

LEITE, K. D. C.; VELANI, V. Divertindo-se com a química: o ensino e a aprendizagem por meio do lúdico. Braz. J. of Develop., v. 5, n. 11, p. 25115-25133, 2019.

MARQUES, J. D. C. et al. Nível Médio Técnico e Cursos de Graduação: comparativo de vagas e ingressantes no Instituto Federal do Amapá, Brasil (20172018). Research, Society and Development, v. 9, n. 8, p. e228985375, 2020. https://rsdjournal.org/index.php/rsd/article/view/5375

RC: 78603

Disponível em: https://www.nucleodoconhecimento.com.br/formazione-it/confronto- 
MORETTO, M.; WITTKE, C. I. Capacidades de linguagem desenvolvidas em estudantes do ensino médio a partir de uma dinâmica de produção de textos focada no ENEM. Diálogo das Letras, v. 7, n. 2, p. 155 - 172, 2018.

PENHA, A. C. F. M. et al. Matrículas da Educação Especial na Educação Profissional Técnica de Nível Médio no Estado do Amapá (2015-2018). Research, Society and Development, v. 9, n. 7, p. e881974867, 2020. https://rsdjournal.org/index.php/rsd/article/view/4867

PONTES, A. N. et al. O Ensino de Química no Nível Médio: Um Olhar a Respeito da Motivação. XIV Encontro Nacional de Ensino de Química (XIV ENEQ) Curitiba PR: Universidade Federal do Paraná 2008.

SILVEIRA, F. L.; STILCK, J.; BARBOSA, M. Comunicações: Manifesto sobre a qualidade das questões de Física na Prova de Ciências da natureza no Exame Nacional de Ensino Médio. Caderno Brasileiro de Ensino de Física, v. 31, n. 2, p. 473-479, 2014.

UFPE. Relação dos Cursos com novos Pesos e Notas Mínimas do ENEM 2019 que serão aplicados no Processo Seletivo UFPE| SiSU 2020. 2021. Disponível em: < https://www.ufpe.br/documents/38970/2199517/Pesos+e+notas+m\%C3\%ADnimas+ ENEM_UFPE+2020_+Mudan\%C3\%A7as+nos+cursos_03.06.19.pdf/85759192-f4cc4817-8541-026a5e06f7c4 >. Acesso em: 24 fev 2021.

Pubblicato: Marzo 2021

Approvato: Marzo 2021

RC: 78603

Disponível em: https://www.nucleodoconhecimento.com.br/formazione-it/confronto- 